\title{
Functional Integrals and Adiabatic Limits in Field Theory*
}

\author{
S. DESER $\dagger$ \\ Institute for Advanced Study, Princeton, New Jersey \\ (Received March 26, 1955)
}

\begin{abstract}
The behavior of a system of coupled fields as some of the particles become very heavy is investigated. By means of a technique expressing the relevant exact Green's functions as functional integrals, the limiting process need only be performed for bare one-particle propagators to get the result for the exact functions. It is shown that the resultant propagators are those for the remaining finite masses moving in external fields generated by the heavy particles acting as static point sources. The situation in which the coupling to the intermediate fields has no simple nonralativistic limit is also discussed.
\end{abstract}

\section{I.}

$T$ HE behavior of a coupled system of fields as some of the particle masses are taken much greater than the others is always supposed to approach in such a limit that of a reduced system, in which the heavy particles act as static sources of the fields to which they are coupled. It is the purpose of this note to supply a proof of this adiabatic limiting assumption.

By use of a technique expressing the relevant propagation functions as functional integrals, the desired limiting process need only be performed in one simple part of the exact expressions, to include all radiative corrections and interactions, at once.

We shall, for simplicity, consider the coupling of several fermions via a boson field (of zero or finite mass) and with various (scalar, vector, etc.) couplings. Since the limit considered is essentially one in which the heavy particle becomes nonrelativistic, couplings which have no simple behavior in this limit [such as $p s(p s)]$ present difficulties which will be discussed separately.

\section{II.}

The technique to be employed is one which stems both from Feynman's formulation as path integrals, ${ }^{1}$ and Schwinger's functional derivative equations ${ }^{2}$ for the propagators in field theory, and expresses them as functional integrals over all possible prescribed external boson field distributions.

We shall not here derive the basic expressions for Green's functions, since an independent derivation (for the case neglecting vacuum polarization effects) has already been published. ${ }^{3}$ Concerning ourselves first with this approximate case, we may write for the Green's function for one fermion interacting with a boson field

* An abstract of some of this work was presented at the Pacific Northwest American Physical Society meeting, July, 1954 [Phys. Rev. 96, 843A (1955)].

$\dagger$ F. B. Jewett Fellow.

1 R. P. Feynman, Phys. Rev. 80, 440 (1950); 84, 108 (1951)

2 J. Schwinger, Proc. Natl. Acad. Sci. U. S. 37, 452 (1951).

3 S. F. Edwards and R. E. Peierls, Proc. Roy. Soc. (London) A224, 24 (1954). and in the presence of an external, prescribed field $\phi$,

$$
\begin{array}{r}
G_{+}^{\prime}\left(x, x^{\prime} ; \phi\right)=\frac{1}{N} \int G_{+}\left(x, x^{\prime} ; \Omega\right) \\
\quad \times \exp \left[-\frac{1}{2} i \int(\phi+\Omega)(y) \Delta_{+}^{-1}\left(y, y^{\prime}\right)\right. \\
\left.\quad \times(\Omega+\phi)\left(y^{\prime}\right)(d y)\left(d y^{\prime}\right)\right] \delta \Omega \\
N=\int \exp \left[-\frac{1}{2} i \int \Omega \Delta_{+}{ }^{-1} \Omega(d y)\left(d y^{\prime}\right)\right] \delta \Omega
\end{array}
$$

where $\Delta_{+}$is the Green's function for the boson field (which may have finite or zero mass).

$G_{+}$satisfies the equation of a bare particle in an external field $\Omega$ :

$$
(\gamma p+m+g \Gamma \Omega) G_{+}=1 .
$$

$\Gamma$ represents the various elementary couplings, and $\Omega$ may be a vector, pseudoscalar, etc.

It may also be proved that in general, the exact $n$ fermion, $r$-boson Green's function is in this approximation $^{4}$ :

$$
\begin{aligned}
& G_{n, r}{ }^{\prime}\left(x_{1} \cdots x_{n}, x_{1}{ }^{\prime} \cdots x_{n}{ }^{\prime} ; \eta_{1} \cdots \eta_{r} ; \phi\right) \\
& =\frac{i^{[r / 2]}}{N} \int G_{+}\left(x_{1}, x_{1}{ }^{\prime} ; \Omega\right) \cdots G_{+}\left(x_{n}, x_{n}{ }^{\prime} ; \Omega\right) \Omega\left(\eta_{1}\right) \cdots \Omega\left(\eta_{r}\right) \\
& \quad \times \exp \left[-\frac{1}{2} i \int(\phi+\Omega) \Delta_{+}{ }^{-1}(\phi+\Omega)(d y)\left(d y^{\prime}\right)\right] \delta \Omega,
\end{aligned}
$$

suitably antisymmetrized in the fermion variables. Thus, all effects of interaction and radiative corrections are taken care of in the sum over possible distributions of virtual quanta (as represented by the external field $\Omega$ ), weighted by exponential, the action integral for the boson field.

Equation (3) for $r=0$ follows directly from the functional differential equations for $G_{n}$, for example, as

${ }^{4}$ Inner products are always understood between the vectors $\Gamma$ and $\Omega$, and with the tensor $\Delta^{-1}$. 
given in reference 2 . That is, just as the result (1) arose from the formal integration of the equation

$$
[\gamma p+m+g \Gamma(\langle\phi\rangle-\delta / \delta J)] G_{+}^{\prime}=1 \text {, }
$$

integration of the analogous one for $n$ fermions,

$$
\begin{array}{r}
{\left[\gamma_{1} p+m+g \Gamma_{1}(\langle\phi\rangle-\delta / \delta J)\right]_{1} G_{n}{ }^{\prime}\left(x_{1} \cdots x_{n}, x_{1}{ }^{\prime} \cdots x_{n}{ }^{\prime}\right)} \\
=G_{n-1}{ }^{\prime}\left(x_{2} \cdots x_{n} ; x_{2}{ }^{\prime} \cdots x_{n}{ }^{\prime}\right)
\end{array}
$$

yields the desired result. Remembering that to include $r$ mesons, ${ }^{5}$ one must apply the operator $\delta / \delta J=\Delta_{+} \delta / \delta \phi r$ times to $G_{n, 0}$, the result follows for that case as well.

Perturbation theory emerges immediately if one inserts the Born approximation series for the $G_{+}$'s, and integrates the resultant expressions formally.

The value of the formulation just given for our purpose is apparent. The mass parameters appear only in the $G_{+}$'s, that is, bare particle Green's functions. There a limit such as $m \rightarrow \infty$ may be taken very easily. To illustrate the general proof, consider the two-body problem, ${ }^{6}$

$G_{+12}\left(x_{1} x_{2}, x_{1}^{\prime} x_{2}^{\prime} ; 0\right)$

$$
\begin{aligned}
& =(1 / N) \int G_{+}\left(x_{1}, x_{1}{ }^{\prime} ; \Omega\right) G_{+}\left(x_{2} x_{2}{ }^{\prime} ; \Omega\right) \\
& \quad \times \exp \left[-\frac{1}{2} i \int \Omega \Delta_{+}{ }^{-1} \Omega(d y)\left(d y^{\prime}\right)\right] \delta \Omega .
\end{aligned}
$$

Let us suppose $m_{2}$ very large; then $G_{+}\left(x_{2}, x_{2}{ }^{\prime} ; \Omega\right)$ $=\left(x_{2}\left|\left(\gamma p+m_{2}+g \Gamma \Omega\right)^{-1}\right| x_{2}^{\prime}\right)$ can be given explicitly for couplings with nonrelativistic limit $\left(\Gamma=1, \gamma_{\mu}\right)$. The solution of the limiting equation,

$$
\left(p_{0}-m_{2}-g \Omega\right) G_{+}=1 \text {, }
$$

is

$$
\begin{aligned}
G_{+}{ }^{\prime}(2)= & -i \delta\left(r_{2}-r_{2}{ }^{\prime}\right) \theta\left(t_{2}-t_{2}{ }^{\prime}\right) \\
& \times \exp \left[-i m_{2}\left(t_{2}-t_{2}{ }^{\prime}\right)-i g \int_{t_{2}^{\prime}}^{t_{2}} \Omega\left(r_{2}, t\right) d t\right], \\
\theta(x)= & \begin{cases}1, & x>0 \\
0, & x<0\end{cases}
\end{aligned}
$$

Setting this into Eq. (6), absorbing the phase factor $\exp \left[-i m_{2}\left(t_{2}-t_{2}^{\prime}\right)\right]$ into the definition of $G_{+12}$, and

\footnotetext{
${ }^{5}$ S. Deser and P. C. Martin, Phys. Rev. 90, 1075 (1953).

${ }^{6} G_{+}$can also be written symbolically as an ordered proper-time integral, $G_{+}=\int d s \exp [-s(\gamma p+m+g \Gamma \Omega)]$, and so $G_{+12}$ could be represented as a double integral of this sort; however, this does not make solution any simpler.
}

letting the source times get very large,

$$
\begin{gathered}
G_{+12} \rightarrow \exp \left[\frac{1}{2} i g^{2} Y(0)\left(t_{2}-t_{2}{ }^{\prime}\right)\right](1 / N) \int G_{+}\left(x_{1}, x_{1}{ }^{\prime} ; \Omega\right) \\
\times \exp \left\{-\frac{1}{2} i \int\left[\Omega(x)+g Y(r) \delta(t) \delta\left(r-r_{2}\right)\right] \Delta_{+}{ }^{-1}\right. \\
\left.\times\left[\Omega\left(x^{\prime}\right)+g Y\left(r^{\prime}\right) \delta\left(t^{\prime}\right) \delta\left(r^{\prime}-r_{2}\right)\right](d x)\left(d x^{\prime}\right)\right\} \delta \Omega, \\
Y(r)=e^{-\mu r} / r \text { or } 1 / r .
\end{gathered}
$$

The $\exp \left[\frac{1}{2} i g^{2} Y(0)\left(t_{2}-t_{2}{ }^{\prime}\right)\right]$ factor outside represents the well-known self-energy of a static source, and is also an irrelevant phase factor; comparing this expression with Eq. (1), we see that particle 1 moves in an effective static external field $\phi=Y\left(r-r_{2}\right)$, or otherwise stated, it propagates in a region with a static point source (current) located at $r_{2}$. This proof is of course the same for $G_{n, r}$ in the limit as any of the $n$ particles become heavy and are therefore representable as fixed $\delta$-function currents. In particular, had we taken in our illustration $m_{1}$ going to infinlty as well, we would have obtained the old result for the energy of a system of two fixed sources exchanging bosons. In this trivial limit, the functional integration is immediately performed. The energy consists of a self term $\frac{1}{2} g^{2} Y(0)$ for each particle, and an interaction term $g_{1} g_{2} Y\left(r_{1}-r_{2}\right)$.

The cases discussed in the foregoing were of a type for which the coupling approached a definite nonrelativistic limit. In the case of $\gamma_{5}$ or $\gamma_{5} \gamma_{\mu}$ coupling the situation is more complicated. At first glance, the heavy particle no longer interacts with the field in the limit, since a large component reduction of $\gamma_{5}$ or $\gamma_{5} \gamma_{\mu}$ brings a $1 / m_{2}$ factor into the coupling, and the equation for $G_{+}$reduces to the form

$$
\left(p_{0}-m-g \frac{\boldsymbol{\sigma} \cdot \nabla}{m} \phi\right) G_{+}=1 .
$$

A new coupling constant $f=g / m_{2}$ may be defined, of course, and if taken finite in the limit, the usual gradient coupling arises, so that the heavy nucleon acts as a source of the form ${ }^{7} \boldsymbol{\sigma} \cdot \nabla \delta\left(r_{2}\right)$, in the weak coupling approximation, where noncommutativity of the $\sigma$ 's is neglected.

However, the equation ${ }^{8}$

$$
\left(p_{0}-m-g \gamma_{5} \phi\right) G_{+}=1
$$

${ }^{7}$ The singularity of the potential obtained, is, of course, due to the passage to the state limit. Inclusion of recoil reduces the singularity; see for example, L. Van Hove, Phys. Rev. 75, 1519 (1949).

${ }^{8}$ An alternate procedure is to perform a Foldy transformation on Eq. (11), and then do the functional integrals. 
may be solved directly, and since $\gamma_{5}^{2}=-1$, we have

$$
\begin{aligned}
& G_{+}=-i \delta\left(r-r_{2}\right) \theta\left(t_{2}-t_{2}{ }^{\prime}\right) \\
& \times \exp \left[-i m\left(t_{2}-t_{2}{ }^{\prime}\right)-i \gamma_{5} g \int \Omega\left(r_{2}, t\right) d t\right] \\
&=-i \delta\left(r-r_{2}\right) \theta\left(t_{2}-t_{2}{ }^{\prime}\right) \exp \left[-i m\left(t_{2}-t_{2}{ }^{\prime}\right)\right] \\
& \times\left[\cos i \int g \Omega-\gamma_{5} \sin i \int g \Omega\right],
\end{aligned}
$$

and the first part remains in the limit to act as effective source of a field resembling the scalar one. ${ }^{9}$ The $\cos i \mathcal{S} g \Omega$ term may be rewritten as $\frac{1}{2}[\exp (g S \Omega)+\exp (-g \mathcal{S} \Omega)]$. The first part alone is the scalar result, while the second half, which will in general also contribute, represents a source at the same point, but with opposite charge. Thus, the propagator for the remaining particles is a superposition of two such terms. If we consider the special case of the $G_{+}{ }^{\prime}$ of particle 2 by itself, then, since the boson action is even, the two terms have the same integral, and all the results are as in the scalar case. This is not surprising, since the field effects can only enter in even powers in the nucleon propagator, and since only the self-field exists, $\phi_{s}{ }^{2}$ and $\left(i \gamma_{5} \phi_{p s}\right)^{2}$ are of course identical. In the many-particle Green function, however, there are other meson field sources around; while the above remarks still hold for those effects which are even as well in the other $\Omega$ 's, it is no longer the case when odd terms from other particles enter. Then the parity counts and the superposition results.

\section{III.}

We turn now to the complete problem, including closed loops. In this case, the action function of the boson field is not so simple; the $\Delta_{+}{ }^{\prime}$ is itself a functional of $\Omega$, and in fact satisfies equations:

$$
\left(k^{2}+\mu^{2}\right) \Delta_{+}^{\prime}=1+i g \underset{\delta J}{\delta} \operatorname{tr}\left(\sum \Gamma_{i} G_{+i}{ }^{\prime}\right),
$$

the sum extending over the various fermion fields. It is to be expected that our results still remain, since the heavy particles become more and more difficult energetically to create and therefore their closed loops will not appear. In fact, since, as we have seen, the limiting behavior for $G_{+}[\phi]$ is one in which negative-energy states vanish, there can be no heavy pair creation.

\footnotetext{
${ }^{9}$ Similar results have been obtained previously by J. V. Lepore, Phys. Rev. 87, 209 (1952).
}

Thus, in the limit as $m \rightarrow \infty$, the vacuum polarization effects due to the heavy particles disappear and they merely act once again as static point sources of the field. However, the field thus generated is no longer the simple $Y$, but one modified by the vacuum polarization of the finite-mass particles, that is, $\phi(x)=\int_{-\infty}{ }^{\infty} \Delta_{+}{ }^{\prime}\left(x-x_{2}\right) d t_{2}$, where $\Delta_{+}{ }^{\prime}$ satisfies Eq. (13), but omitting the effect of the heavy field. Of course, the complete problem with closed loops can only be stated implicitly in closed form; that is, while an expression for $G_{+}$can be written down, it is purely formal, being actually a transcendental functional integral equation rather than the much simpler Eq. (1).

Thus, we may still write

$$
G_{+}^{\prime}[0]=\int \delta \Omega G_{+}(\Omega) \exp \left[-\frac{1}{2} i \int \Omega T \Omega(d y)\left(d y^{\prime}\right)\right]
$$

$T$ itself depends on $G_{+}$and is defined through

$$
\begin{aligned}
& \exp \left[-\frac{1}{2} i \int \Omega T \Omega(d y)\left(d y^{\prime}\right)\right] \\
& \quad=\int \delta J \exp \left[i \int J \Omega(d y)+i \int^{\phi} \Delta_{+}^{-1}\left[\phi^{\prime}\right] \phi^{\prime} \delta \phi^{\prime}\right]
\end{aligned}
$$

and $\phi=\int^{J} \Delta_{+}\left[J^{\prime}\right] \delta J^{\prime}$, which reduces to the usual result when $\Delta_{+}$is independent of $\phi$.

In this form, the parameter $m$ enters in a fashion which precludes any proof as to the limiting behavior of $G_{+}{ }^{\prime}$. However, as was stated above, it is evident that there can be no vacuum polarization of the heavy field, since it loses its negative-energy components. ${ }^{10}$ Alternatively, had we written the vacuum polarization contribution in the form $G_{+}^{\prime}[0]=\int \delta \Omega G_{+}(\Omega) \exp \left[-\frac{1}{2} i\right.$ $\left.\times \int \Omega \Delta_{+}{ }^{-1} \Omega(d y)\left(d y^{\prime}\right)-i L^{\prime}(\Omega)\right]$ where $\exp \left[-i L^{\prime}(\Omega)\right]$ is the contribution from closed loops, and used the formal Fredholm series result, say, for $\exp \left[-i L^{\prime}\right]$, we would have observed that each term vanishes due to the $\theta(t)$ factor in the $G_{+}[0]$ to be inserted in the series. $\theta(t)$, of course, just states that negative energy states have been dropped.

I wish to thank Dr. Robert Oppenheimer and the Institute for Advanced Study for their hospitality, and Dr. R. L. Arnowitt for interesting conversations.

${ }^{10} \mathrm{Had}$ the $\gamma_{0}$ been kept in Eq. (11), negative energy states would have entered, but the equation could not then be solved. However, with "relativistic" couplings, the question whether one or another reduction is the more accurate only has meaning with reference to the full recoil solution. 